\title{
Improving the performance parameters of railway wheels with the help of optimal design technologies of their electric pulse processing
}

\author{
Igor Vakulenko ${ }^{1}$, Nickolay Kuzin $^{2,3, *}$, Leonid Vakulenko $^{4}$, Sergey Raksha $^{1}$, and Svetlana Proidak $^{1}$ \\ ${ }^{1}$ DNURT, Department of Applied Mechanics and Materials Science,49010,Dnipro,Lazaryan St., 2, Ukraine \\ ${ }^{2}$ DNURT, Lviv Branch, 79052, Lviv, I.Blazhkevych Str., 12-a, Ukraine \\ ${ }^{3}$ Lviv Research Institute of Forensic Science, 79000 Lviv, Ukraine \\ ${ }^{4}$ Head Department of the Pridniprovk Railway, 49600, Dnipro, D.Yavornizkogo Av., 108, Ukraine
}

\begin{abstract}
The processing by pulses of electric current (PEC) of a fragment of the rim of a railway wheel was carried out on the DS10D equipment. When the density of the electric current is from 3 to $17 \mathrm{~A} / \mathrm{mm}^{2}$, the processing cycle consisted of the action of a pulse with a duration of 2.5-3 $\mathrm{s}$ and a pause between pulses of $4 \mathrm{~s}$. After 150 cycles of PEC, the hardness of cold-formed metal was reduced from 10 to $20 \%$. Studies of the parameters of the metal structure of the wheels have determined that the processing of PEC leads to a decrease in the number of defects in the internal structure, which are accumulated as a result of cold deformation of the rim along the rolling surface of the railway wheel. It has been established that, according to the nature of the influence on the hardness distribution of carbon steel, the PEC treatment corresponds to changes during tempering in the average temperature range and allows to significantly increase the operating parameters of the wheels of railway equipment.
\end{abstract}

\section{Introduction}

Compared to the thermal softening technology of hardened metal, processes that are based on the use of other physical influences such as magnetic field processing [1] or electric current pulses [2] have received sufficient propagation. Combines such processing with the absence of significant thermal effects on the development of processes structural transformation.

Known effects of plasticization from the action of electric current pulses are associated with the influence on the motion cloud free electrons in the crystalline lattice of a metal material [3]. As the energy of moving electrons increases as a result of the electric current, their impulse is partially transmitted to the dislocations, resulting in a lowering of the activation energy for the start of displacement [4]. However, according to [5], the effect plasticization of metal materials is considered not be related with activation energy, but depends on the degree of divergence between the direction of action electric impulse and the propagation plastic deformation of the metal.

Taking into account that in the nodes of crystal lattices there are non-neutral metal atoms, and ions, a local violation in their arrangement, for example, in the formation of a dislocation, leads to the idea that the dislocation must also have a certain electric charge. Thus, the interaction of the dislocation with the purposeful movement of the cloud moving electrons should be based on the influence of electric pulse on effect plasticization of the metal material.

\section{Status of the problem}

The origin and distribution of the plastic flow in metals and alloys is accompanied by the movement of dislocations with different signs, which are characterized by a different reaction to the action of electric pulse [6]. Under these conditions, with a predetermined direction in relation to the active stress at the sample load, the summation of the pulses electric current will lead to acceleration of the dislocations one sign and complicate the motion of dislocations opposite sign. For dislocations, which is characterized by a relatively low resistance level by the obstacle, the pulse of electric energy will be sufficient to start their movement. As a result, a local acceleration of a number dislocations of such a sign will occur, which in turn will lead to decrease in the operating syress to ensure the conditions for the continuous propagation of plastic deformation [7]. For dislocations of the opposite sign, the separation from the blocking places can only be due to the occurrence of thermal fluctuations of a certain level. This is due to fact that the nature of effect pulse action 
electric current is an obstacle to the beginning of motion such dislocations. The ratio between the quantities of indicated dislocations and causes achievement the effect of plasticization metal on the action of pulses electric current. At the same time, the sensitivity of metal material to the direction of action of the pulses should be associated with the recombination of the dislocation structure, which will lead to an appropriate directional plastic deformation.

Taking into account that the first attempts to use the pulse action of electric current were aimed at increasing the plastic propertiesmonocrystalsof metals during plastic deformation, the electric current density in the pulse was equal to $10^{3}-2 \cdot 10^{3} \mathrm{~A} / \mathrm{mm}^{2}$. Approximately similar regimes were used in the treatment of cold drawn wire for increase its plastic properties [2-4]. The use of this technology was not limited only to the increased plasticity of cold drawn metal, it was found to be used with special surface finishes, coatings, etc. [8-10]. The high density of electric current in a pulse restricts the use of such technology, primarily the power of equipment. Indeed, according to $[5,6]$, the intersection of single crystals and cold drawn wire basically did no more $1 \mathrm{~mm}$. The use of processing metal layers on the rim railway wheel, based on the action of electric current with such a high density in the pulse is limited to the equipment required power.

\section{Material and methodology of research}

The material for research was the fragment of rim railway wheel after operation with a carbon content of $0.61 \%$ and concentration of other chemical elements complied with the requirements of the Standard of Ukraine. The processing of electric current pulses of a fragment rim railway wheel was carried out on the equipment DS10D, using an alternating current.

The calculation of the pulse frequency was carried out under conditions where the thickness of the metal layer is limited to the appearance of the "skin effect" by the ratio:

$$
f_{k}=\left(\pi \cdot \mu_{0} \cdot \mu \cdot \gamma \cdot \delta^{2}\right)^{-1}
$$

where $f_{k}$ - the critical frequency $(\mathrm{Hz})$ above which the "skin effect" occurs, $\gamma$ - the specific electrical conductivity $(\mathrm{Ohm} / \mathrm{m}), \mu_{0}, \mu$ - the magnetic permeability of the vacuum and the test metal, respectively $(\mathrm{Gn} / \mathrm{m}), \delta$ - the thickness of the metal layer that is required process $(\mathrm{m})$

In developing the processing technology of electric current pulses fragment rim railway wheel determined the optimal shape of the pulse electric current, which looked like a symmetric trapezoid. The time interval when increasing and decreasing the amplitude value of the electric current was $0.5-1 \mathrm{~s}$. For the treatment of a layer metal in the thickness of $10-15 \mathrm{~mm}$ there was enough current density to $17 \mathrm{~A} / \mathrm{mm}^{2}$, the processing cycle consisted of a pulse duration of $2.5-3 \mathrm{~s}$ and a pause between them up to $4 \mathrm{~s}$.
The hardness used was measured using the Vickers method ( $H v)$ as a strength characteristic. The evaluation of the parameters thin crystalline structure was carried out using X-ray structural analysis techniques. The microstructure of the metal was studied under a light microscope used methods of quantitative metallography.

\section{Results and discussions}

The metal on the rolling surface rim of the railway wheel during operation is subject to a very high degree of cold plastic deformation with considerable heterogeneity in its distribution. For ease of research, the rolling surface of the railway wheel was divided into three sections, with varying degrees of cold plastic deformation. The plot (I) - part of the rim near the crest, (II) - middle part of the rolling surface and (III) - near the lateral surface of the wheel. Measurement of hardness on the rolling surface confirmed the existence of a significant difference in the degree deformation of the metal rim railway wheel after operation. For regions (I) and (III), the hardness was in the range of 5 5,5 GPa, and for (II) it was $4-4,9 \mathrm{GPa}$. The lower level of hardness on the site (II) is due to the acceleration process of softening cold-deformed metal from heating during the braking stages of the rolling stock. Indeed, if we assume that the area (II) is characterized by a maximum wear metal, then the degree of plastic deformation should be higher compared with other sections of the rim. The results of studies [11] determined that in the case of heating to certain temperatures, the degree of softening metal will be proportional to the value of cold plastic deformation.

Given that the process of electric current pulses is accompanied by changes in the geometric dimensions of the sample (Fig.1) the nature of the influence on the hardness metal may be similar to that of a reversible load $[4,5]$.

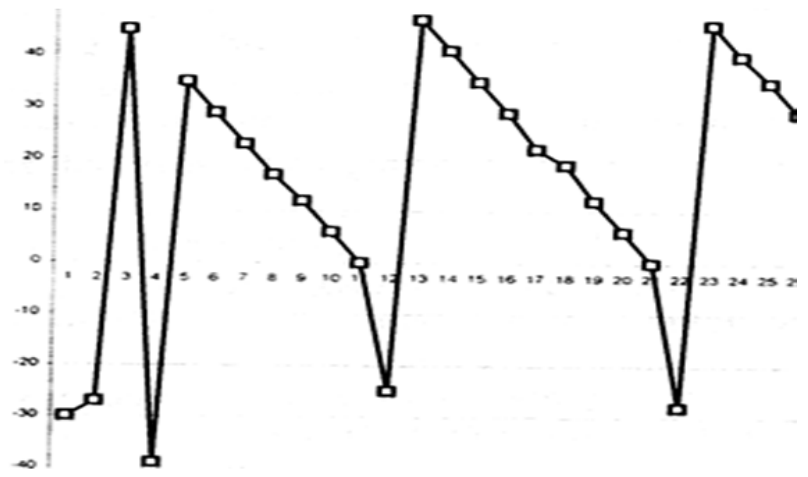

Fig. 1. The sample size change from the cycle number during the processing by pulses of electric current [12].

Indeed, regardless of the nature metal materials, the development of softening processes is observed for a wide range values of plastic deformation, in the event of divergence in the direction or form subsequent loading of the previous one. On the basis of this it can be assumed that in addition to the influence of electric current pulse on the acceleration of motion of cloud free electrons, one should expect the emergence of additional lever in the form of a mechanical component of the process. On the basis of the analysis obtained results, it 
was determined that as a result of the electric current pulses, a certain level of metal softening was achieved. Reduction of the hardness level for the region (I) was 20; for (II) 9 and for (III) $11 \%$ relative to the state after a cold plastic deformation [12]. Taking into account that in electric pulse processing the temperature of the metal did not exceed $50^{\circ} \mathrm{C}$, the achieved level of softening can't be related to the thermal nature of the effect. More it is known that heating up to temperatures of $200-250^{\circ} \mathrm{C}$, due to the development of processes of deformation aging, should lead to an increase in the hardness of colddeformed carbon steels [11]. The obtained of equal effect softening metal on rolling surface railway wheel after electric current pulses is confirmed by the appearance of qualitative changes in the internal structure of the metal.

Figure 2 shows the microstructure of metal volumes near the rolling surface railway wheel after operation. The structure corresponds to the metal after a high degree of cold plastic deformation.

In the background of high turbulence structure colonies of perlite (Fig. 2a,b), the presence sites of structurally free ferrites (Fig. 2c) can be regarded as evidence of heating metal near the rolling surface to temperatures higher than the beginning of phase transformations. On the other hand, the absence in this section signs formation of additional boundary sections, which are characteristic of the development processes of recrystallization [11], indicates a very short period of exposure metal at these temperatures $[12,13]$.

After electric pulses treatment fragment of the rim railway wheel, reducing the level of hardness is accompanied by quite logical changes in the internal structure of the metal (Fig. 3). From the comparative analysis of the structural components, according to external features, the effect of electric pulses should be attributed to the thermal influence on metal after plastic deformation (Fig. 3a). Indeed, in comparison with the structural state metal wheels after operation (Fig. 2a,b), electric pulse treatment results not only in the coarsening of structural components (Fig. 3a,b), but also in some changes in their distribution.

Comparative analysis of sites structurally free ferrite up to (Fig.2c) and after electric pulse treatment (Fig. 3c), confirms the appearance of qualitative differences in the structure of steel. One of them is the emergence of more distinct additional boundaries in volumes of a structurally free ferrite (designation "a", Fig. 3c).The presence in volumes of structurally free ferrite distorted lines, similar to the lines slipping of dislocations, indicates a high degree of cold deformation with the obligatory change in the direction of loading.

The action of pulses of electric current is accompanied by the appearance of additional distortions, which significantly distinguishes them from the lines of slippage in the propagation of plastic flow in metallic materials [11].Thus, we can assume that degree of softening obtained from the action of pulses electric current in reality is accompanied by significant changes in the internal structure of the metal rim after cold plastic deformation.

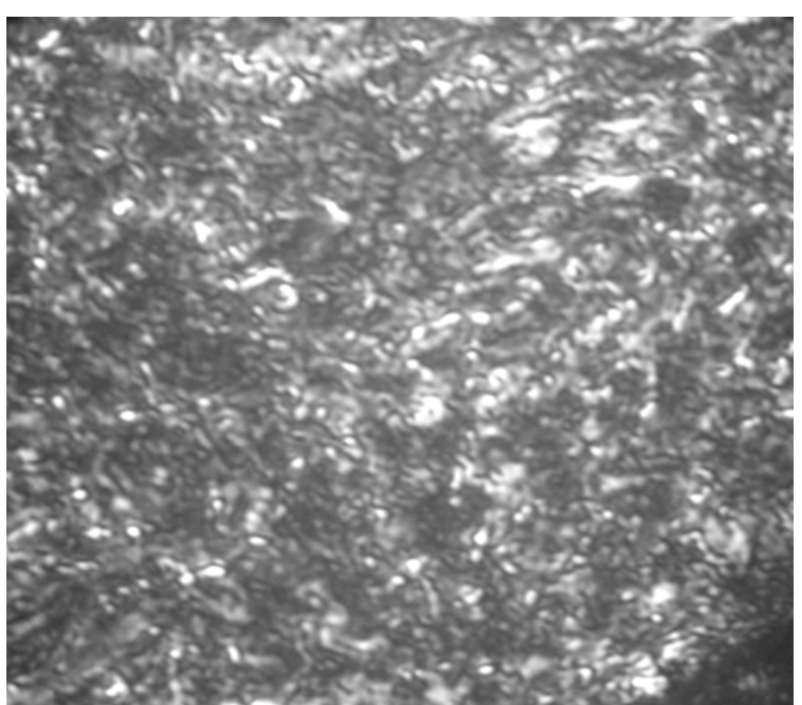

a

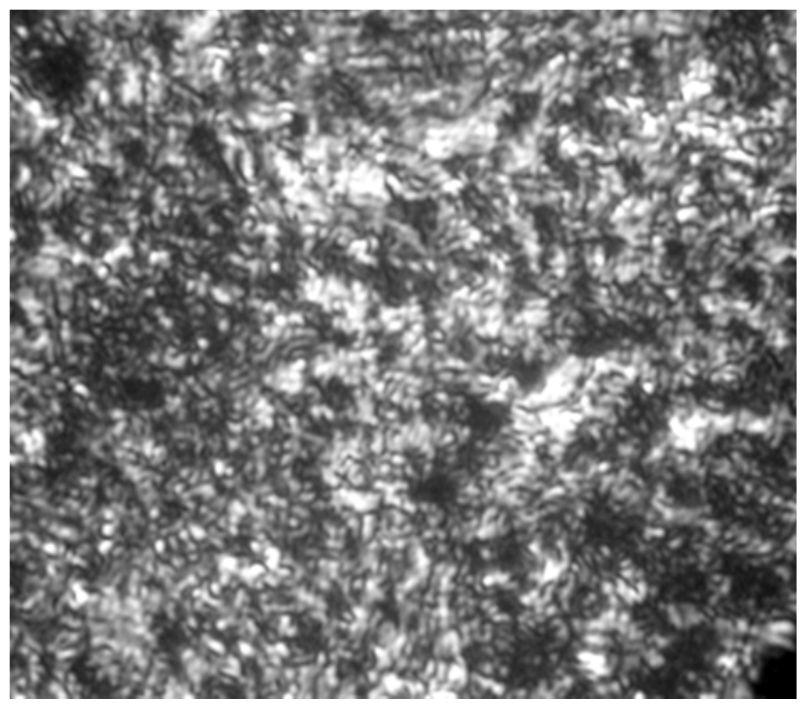

$\mathrm{b}$

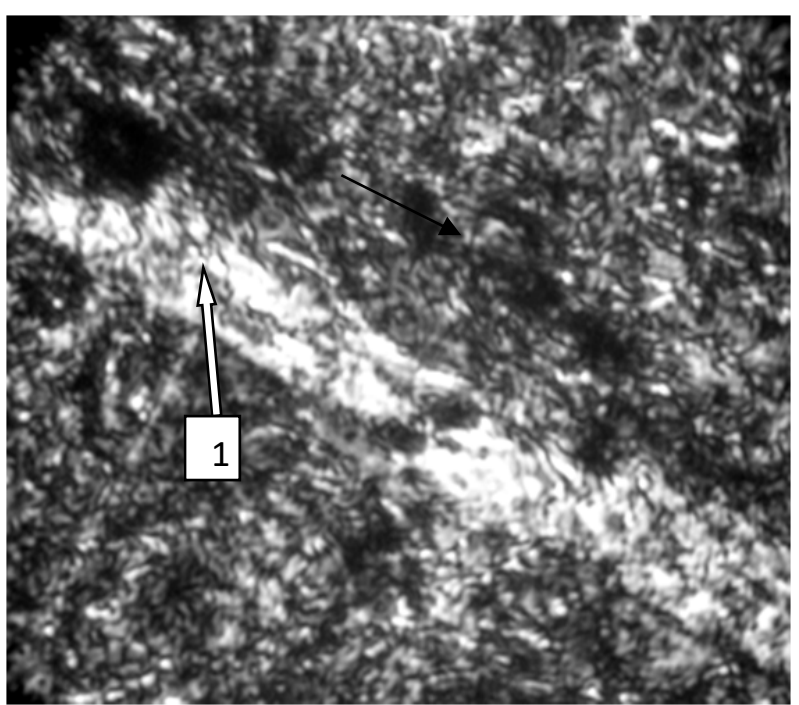

$\mathrm{c}$

Fig.2. Microstructure of the steel near the rolling surface of the wheel after decommissioning. Pearlite colonies $(\mathrm{a}, \mathrm{b})$ and site of structurally free ferrite - (1) (c), magnification 250. 


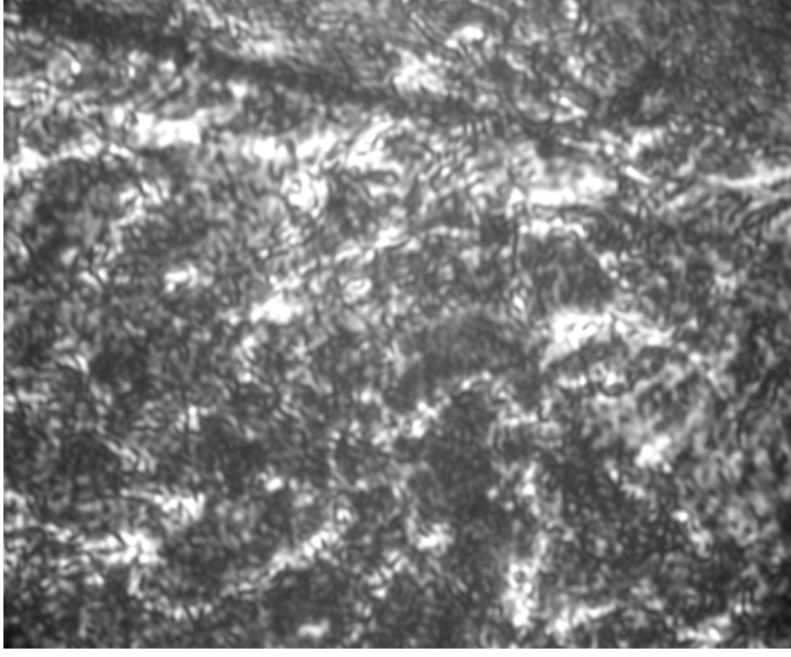

$\mathrm{a}$

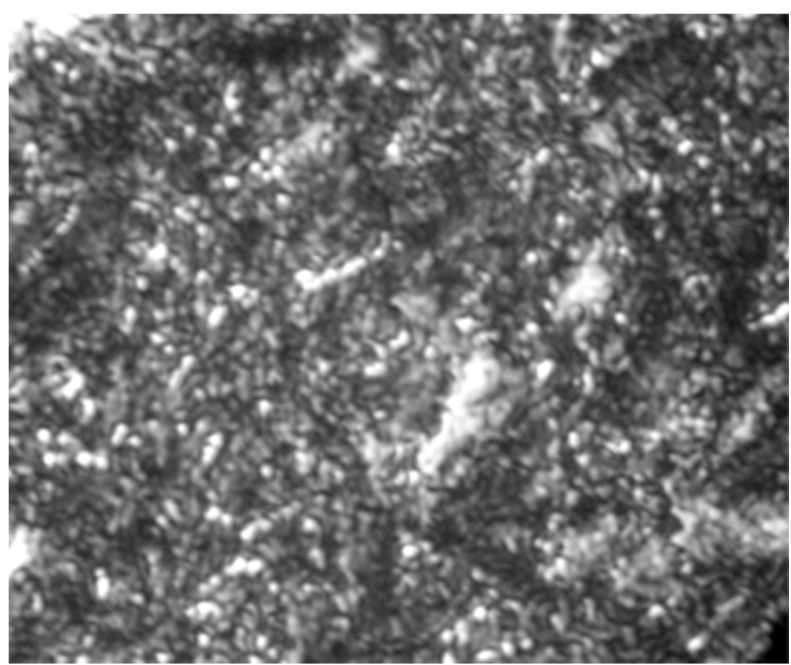

b

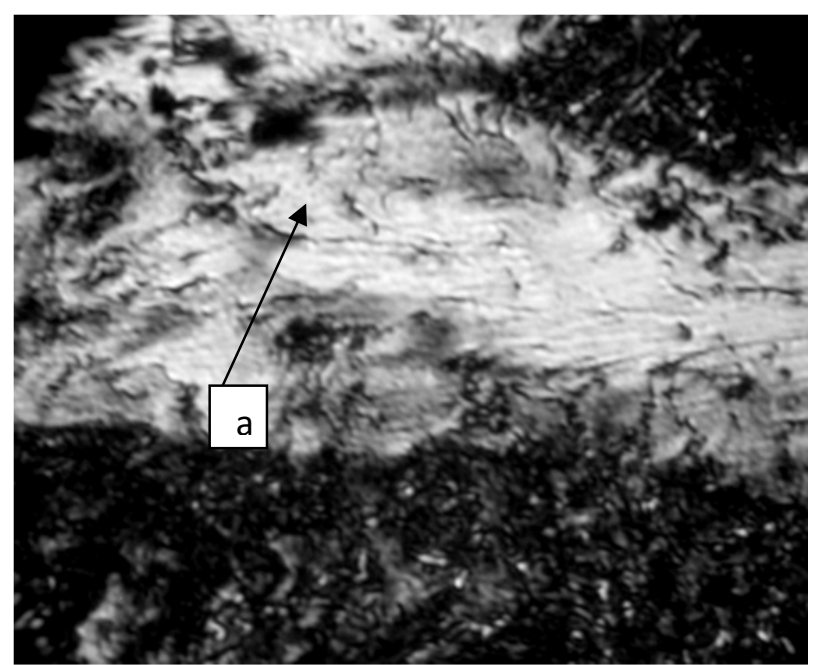

c

Fig.3. The microstructure of the steel near the rolling surface of the wheel after the treatment of the IES (a, b - perlite colonies, $\mathrm{c}-$ a section of structurally free ferrite), magnification 250 .

Considering the mechanism of development process of softening in the cold deformed metal from the action of pulses electric current, the cyclic change in the size of the metal specimen undergoing processing becomes a definite value. Indeed, in the conditions of a cyclic loading, the micro volume of a metal is subject to the action of a definite total deformation. However, under the influence of the emerging internal stresses, the most soft structural components will first be deformed. As a result development of processes, the relaxation of internal stresses in volumes of a structurally free ferrite, the magnitude existing stresses may not be sufficient for the start deformation of pearlite colonies.

Given the very small change in the geometric dimensions of the specimen (Fig.1) with ferrite hardness about 2 times below the pearlite colony, a large part of the plastic deformation should be concentrated in the regions of a structurally free ferrite of steel. According to stoichiometry, in steel of railway wheels, the share of structurally free ferrite can reach up to $20-25 \%$.

Assuming that the main effect of action pulses electric current is concentrated in ferrite, the additive contribution to the general effect of softening at the level of $10-15 \%$ is fully justified..

The results of the softening metal rim railway wheel on the rolling surface after deformation were confirmed by X-ray structural analysis (Table). The analysis of the results on the measurement of the internal stresses $(\sigma)$, density of the dislocation by the interference (211) - ( $\left.\rho_{211}\right)$, distortions of the second kind $(\mu)$ and the size of the coherent scattering regions $(D)$, regardless studied areas of the wheel (I, II, III) development of very complicated by the nature of structural transformations in the metal under the influence of pulses electric current.

Table. Changing of parameters fine crystalline structure of the ferrite from pulses electric current.

\begin{tabular}{|l|c|c|c|}
\hline \multirow{2}{*}{ Parameter } & \multicolumn{3}{|c|}{ Number areas of rim railway wheel } \\
\cline { 2 - 4 } & I & II & III \\
\hline$\sigma, G P a$ & $-0,064$ & $-0,128$ & $+0,210$ \\
\hline$\rho_{211} \times 10^{11} \mathrm{~cm}^{-2}$ & $3,6 / 2,8^{*}$ & $3,6 / 2,2^{*}$ & $3,9 / 3,0^{*}$ \\
\hline$\mu, \times 10^{-3}$ & $1,09 / 0,84^{*}$ & $1,22 / 0,87^{*}$ & $1,18 / 0.97^{*}$ \\
\hline$D, n m$ & $28,9 / 32,7^{*}$ & $27,7 / 33,0^{*}$ & $27,7 / 31,6^{*}$ \\
\hline
\end{tabular}

Given the very complex conditions of loading the railway wheel on the rolling surface (heterogeneous hardened from cold plastic deformation, rapid heating and cooling metal to different temperatures during braking of rolling stock, etc.), an attempt was made to assess the efficiency of pulses electric current treatment compared to thermal technology.

The figure 4 shows effect of temperature heating on the hardness steel of a railway wheel after cold plastic deformation with a different dispersion of perlite. At the conditions of equal degree of plastic deformation, the hardness of metal increases in proportion to as cending dispersion of the pearlite colony. At the same time, for equal degree of plastic deformation, the softening effect is to a large extent determined by the dispersion pearlite colony of the steel. This is due ability of cementite plate- 
like form to be plastically deformed [11]. An increase of the temperature heating will be accompanied by appropriate metal softening. From the comparative analysis it can be determined that the more finely the plate cementite, the degree of softening will be lower (Fig.4).

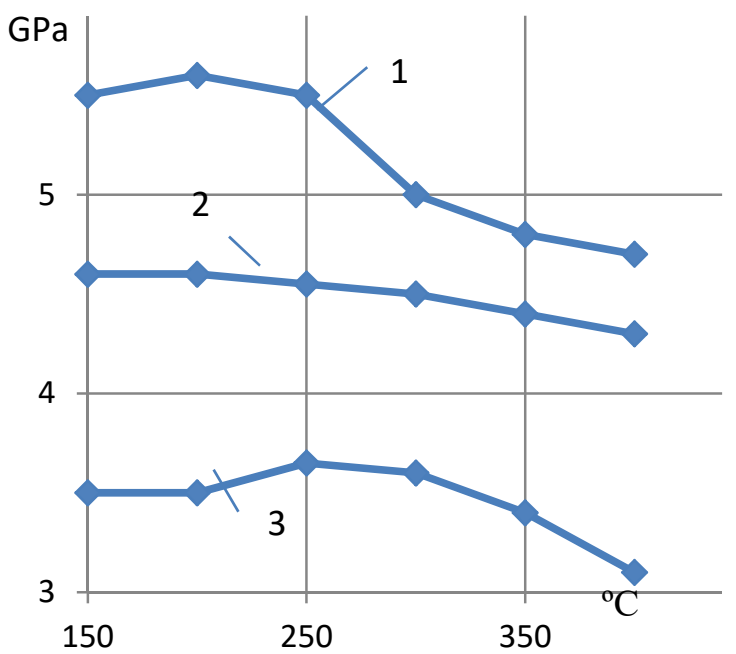

Fig.4. The influence temperature heating on hardness steel of railway wheel after cold deformation (1,3-75, 2-25\%) at high $(1,2)$ and low $(3)$ disperse of pearlite colony.

The nature influence of the degree plastic deformation and dispersion of pearlite colony on the development of softening processes under heating is similar to the known data. So, for the carbon content close investigated steel after cold plastic deformation of $25 \%$, softening at a level of $20 \%$ achieved by heating up to $400^{\circ} \mathrm{C}$, with duration of exposure up to 1 hour. Reduction of hardness by $10 \%$ after cold plastic deformation by $25 \%$ achieved already after the heating of the metal to $300-350^{\circ} \mathrm{C}$. Similar results are obtained and for higher degrees of plastic deformation. Thus, for a steel with a carbon content of $0.7 \%$, deformed by $90 \%$ dragging, the effect of softening by $20 \%$ is achieved after heating to a temperature of $380^{\circ} \mathrm{C}$, and a decrease in hardness by $10 \%$ - by heating up to $300^{\circ} \mathrm{C}$ [11].

Analysis of the experimental data (table) shows that, in general, there is a fairly good agreement both in character and in absolute values with known results [11 13], which should be considered as confirmation of the development of softening processes in carbon steel of wheel fragment after treatment with electric pulses current. Indeed, according results of the study, the process of softening cold-deformed metal is accompanied by decrease in the accumulated number of dislocations, distortions of the second kind and an increase in the size of the regions coherent dispersion (table). At the same time, it is certain interest to evaluate the rate of structural changes depending on the different levels of metal hardening after cold deformation. Taking only the magnitude of the decrease in hardness after treatment pulses electric current $\left(\Delta H_{v}\right)$ as an indicator of the degree metal softening, a formal construction of the degree of change in the characteristics of the finecrystalline structure from (Fig. 5) was carried out. It follows from the presented relationships that, in proportion increase in the effect of metal softening after being treated with electric pulses, decrease in size rate of coherent scattering regions, dislocation density, and second-type distortions is observed.

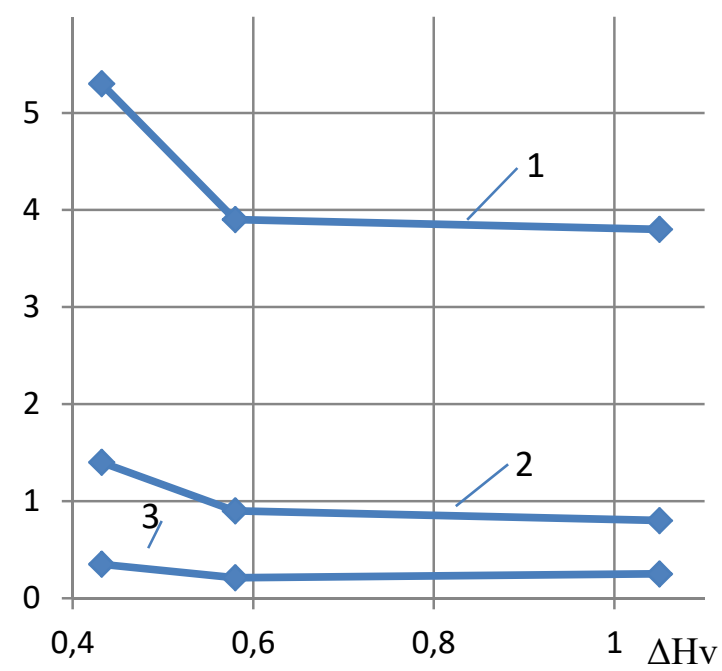

Fig.5. The dependence of change the size coherent scattering regions $(\Delta D, \mathrm{~nm})-(1)$; dislocation density $\left(\Delta \rho_{211}\right.$ $\left.\times 10^{11} \mathrm{~cm}^{-2}\right)-(2)$ and distortions of the second kind $(\Delta \mu$ $\left.\times 10^{-3}\right)$ - (3) of the degree softening $\left(\Delta H_{v}, G P a\right)$ steel after treatment with pulses of electric current.

Although, on the whole, the achievable softening effect from impulse treatment with electric current pulses is proportional to degree strain hardening of metal, the observed change in the sign of residual stresses indicates the development of quite complex structural changes. At the same time, cumulative effect of the non-uniformity of distribution plastic deformation and its directivity on the rolling surface wheel indicates the need to continue studying causes that led to a change in the sign of residual stresses after treatment with pulses electric current.

Thus, if we use the datas of the table and estimate the degree of metal railway wheel softening after treatment of pulses electric current according to the characteristics, compared with the thermal technology, the softening effect may correspond to the heating metal to temperatures of $400-450^{\circ} \mathrm{C}$ [13]. At the same time, the low temperatures of heating metal (up to $50^{\circ} \mathrm{C}$ ) during the processing of pulses electric current can't be attributed development processes of diffusion mass transfer.

In order to determine mechanism of soften colddeformed metal after processing of pulses electric current, an assessment was made of the stresses that caused movement of dislocations from various external influences. Low temperature heating of the metal at processing of pulses electric current immediately excludes the thermal nature of the influence. On other hand, due to high values of dislocation density 
accumulated in crystallographic sliding systems (table), one of the possible mechanisms explaining effect of softening at ambient temperatures may be nonconservative displacement of dislocations under the action of pulses of electric current.

From the balance of forces that determine resistance to displacement dislocation from one slip system to another [14] and facilitate the displacement fragment of the dislocation line into a parallel slip plane [15], the relation obtained:

$$
\operatorname{Sin} \alpha \approx b / h
$$

where $\alpha$ is the angle of disorientation between the line of dislocation and the Burgers vector $(b), h$ is the distance between the parallel sliding planes. Taking into account that $\rho=h^{-2}[16]$, where $\rho$ - the density of dislocations, the relation (2) takes the form:

$$
\operatorname{Sin} \alpha \approx b \sqrt{\rho}
$$

After substituting (3) $b=2,48 \cdot 10^{-7} \mathrm{~mm}$ [11], the experimental values of the dislocation density in accordance with areas rim of railway wheel under study (I, II, III), the calculation shows that as a result of pulses electric current treatment, the angle disorientation between the dislocation line and Biurges vector is reduced. So, for the regions I, II, and III cold-deformed metal, the value $\alpha$ is from $36^{\prime}$ up to $48^{\prime}$ respectively. As a result of the processing of pulses electric current according to the estimated calculations, there was a decrease of the angle of $48 \%$ for the III region, $28 \%$ for II and $17 \%$ for I. The obtained results can be regarded as evidence of a change in the ratio between the edge and screw components of the dislocation structure in the metal rim railway wheel in as a result of processing by pulses of electric current. Thus, one of the explanations softening effect of metal railway wheel as a result the development processes of recombination dislocation structure should be considered. Given that the edge component of the dislocation structure [14] is proportional to the angle, as a result of electrical impulse processing, the part of the screw component should be increased. As a result, the conditions for reducing the metal resistance, of processes creeping dislocations, which should contribute to the development of their annihilation. After processing of pulses electric current, the effect of reducing the hardness of cold-deformed metal on the rolling surface railway wheel practically adequate the heating in the middle temperature range.

\section{Conclusions}

1. Mechanism of softening of cold-deformed metal rim railway wheel after processing by pulses of electric current, based on recombination of dislocation structure. 2. Achieved the effect of softening cold-deformed metal in comparison with thermal technologies, adequate the heating in the middle temperature range.

\section{Acknowledgements}

The authors are grateful to Dr. V.G. Anofriev for providing material for research and Dr. V.A. Sokirko for carrying out the processing of fragment railway wheel by electrical pulses. The authors would like to express their gratitude to the staff of the Department of Applied Mechanics and Materials Science of the University for their participation in the preparation of samples for research.

\section{Literature}

1. M.L. Bernshteyjn., V.N. Pustovoyjt, Termicheskaya obrabotka staljnihkh izdeliyj $v$ magnitnom pole. (Mashinostroenie, 1987).

2. V.I. Spicihn, O.A. Troickiyj, Ehlektroplasticheskaya deformaciya metallov. (Nauka. 1985).

3. A.F. Sprecher, S.L. Mannan, H. Conrad, Acta Met. 34, 1145 (1986).

4. H. Conrad, Materials Science and Engineering A287, 227 (2000).

5. G. Tang, J. Zhang, M. Zheng, et al., Materials Science and Engineering,A. 281, 263 (2000).

6. K F. Yao, J. Wang, M. Zheng, et al., Scripta Materialia, 45.,533 (2001).

7. F. Garofalo, Met. Trans.2, 2315 (1971).

8. K. Ait Aissa, A. Achour, J. Camus, et al.,Thin Solid Films550,264 (2014).

9. S. M. Razavian, B. Rezai, M. Irannajad, et al, Intern. J. of Mining Sci. and Tech., 25.473 (2015).

10. W. L.Morgan,L. A. Rosocha, Chemical Physics of Low-Temperature Plasmas 398, 255 (2012).

11. I. A. Vakulenko, V.I. Boljshakov Morfologiya strukturih $i$ deformacionnoe uprochnenie stali (Makoveckiyj, 2008).

12. L.I.Vakulenko, Problemi koroziyjno-mekhanichnogo ruyjnuvannya, inzheneriya poverkhni, diagnostichnie sistemi: materiali konferencij KMN - 2013, (Fizikomekhanichnogoinstitutu, 2013).

13. L.Vakulenko, S.Myamlin, A.Kovalek. New technologies and achievements in metallurgy, materials engineering and production engineering (Czestochova,.48, 369, 2015).

14. J.Friedel,Dislocations (Pergamon press, 1964).

15. W.T. Read Jr. Dislocations in crystals. (McGraw Hill).

16. A. Guinier, Theorie et Techniquedela Radiocristallographie (Dunod, 1956). 